\title{
Reply: Takotsubo syndrome-induced acute myocardial infarction
}

\author{
Ken Kato $^{1} \cdot$ Yoshiaki Sakai $^{2} \cdot$ Iwao Ishibashi $^{2} \cdot$ Yoshio Kobayashi $^{1}$
}

Received: 5 March 2015/Accepted: 9 March 2015/Published online: 14 March 2015

(C) Springer Science+Business Media Dordrecht 2015

We thank Dr. Madias for his valuable comments on our case report, published online on February 22, 2015 ahead of print in the journal [1]. We would like to respond to his important question about transient attenuation of the voltage of the QRS complexes (AVQRS).

During the acute phase, QRS complexes in leads I and aVL were 4.5 and $1 \mathrm{~mm}$ in amplitude, respectively, under the presence of myocardial edema detected by cardiac magnetic resonance imaging. Five months later QRS complexes in leads I and aVL have been amplified to 6.5 and $2 \mathrm{~mm}$, respectively, corresponding to the complete recovery of wall motion abnormality except infarcted area.

In the present case, mid-ventricular takotsubo cardiomyopathy preceded acute myocardial infarction (AMI). The amplitudes of QRS complexes in leads I and aVL have not been affected by subsequent AMI, because we observed significant ST elevation in leads II, III and aVF on admission as an electrocardiographic change associated with AMI. Therefore, we assume that transient AVQRS in leads I and aVL was possibly caused by mid-ventricular takotsubo cardiomyopathy as suggested by Dr. Madias.

Conflict of interest The authors declare that they have no conflict of interest.

\section{Reference}

1. Madias JE (2015) Takotsubo syndrome-induced acute myocardial infarction. Int $\mathrm{J}$ Cardiovasc Imaging. doi:10.1007/s10554-0150626-x
Ken Kato

kkatou0424@yahoo.co.jp

1 Department of Cardiovascular Medicine, Chiba University Graduate School of Medicine, 1-8-1 Inohana, Chuoku, Chiba, Chiba, Japan

2 Department of Cardiology, Chiba Emergency Medical Center, Chiba, Japan 